\title{
Experimental evidence of tetrahedral interstitial and bond-centered Er in $\mathrm{Ge}$
}

\author{
S. Decoster, ${ }^{1, a)}$ B. De Vries, ${ }^{1}$ U. Wahl, ${ }^{2}$ J. G. Correia, ${ }^{2}$ and A. Vantomme ${ }^{1}$ \\ ${ }^{1}$ Instituut voor Kern- en Stralingsfysica and INPAC, KULeuven, 3001 Leuven, Belgium \\ ${ }^{2}$ Instituto Tecnológico e Nuclear, Estrada Nacional 10, 2686-953 Sacavém, Portugal and Centro de Física \\ Nuclear da Universidade de Lisboa, Avenida Prof. Gama Pinto 2, 1649-003 Lisboa, Portugal
}

(Received 28 May 2008; accepted 17 September 2008; published online 10 October 2008)

\begin{abstract}
We report on an emission channeling study of the lattice site location of implanted Er in Ge together with its thermal stability. We found direct experimental evidence of Er atoms located on the tetrahedral $(\mathrm{T})$ interstitial site and on the bond-centered (BC) site, with a maximum total occupancy after annealing at $400{ }^{\circ} \mathrm{C}$. Whereas Er is expected to occupy the $\mathrm{T}$ site in a diamond crystal structure, the observation of $\mathrm{BC} \mathrm{Er}$ in $\mathrm{Ge}$ is more surprising and believed to be related to the Er-vacancy defect in the split-vacancy complex configuration. (c) 2008 American Institute of Physics. [DOI: 10.1063/1.2996280]
\end{abstract}

During the past decades, the optical properties of Er integrated in semiconductors have been of great interest for photonic applications, especially in $\mathrm{Si}$ since the wavelength of the ${ }^{4} I_{13 / 2} \rightarrow{ }^{4} I_{15 / 2}$ transition of the $\mathrm{Er}^{3+}$-ion corresponds to the minimum absorption of silicabased optical fibers. The properties and dependencies of the luminescence in Er-doped $\mathrm{Si}$ or Si-based materials have been investigated intensively. However, due to the so-called thermal and concentration quenching, its room temperature luminescence yield remains below needs. ${ }^{1}$

In the search for alternative semiconducting host materials, the Ge:Er system received little attention so far because bulk $\mathrm{Ge}$ has a band gap $(0.67 \mathrm{eV})$ that is smaller than the energy corresponding to the technologically interesting optical emission wavelength of Er, i.e., $1.54 \mu \mathrm{m}(0.80 \mathrm{eV})$. This results in a large self-absorption and consequently a very low efficiency. However, by using Ge nanoparticles, it has been possible to tune the band gap with the size of the nanoparticles, and thus to reduce the interband absorption. ${ }^{2}$ The stronger quantum confining effect and a better controlled oxidation with respect to $\mathrm{Si}$, makes it a promising candidate as an Er-host for future photonic applications. ${ }^{3,4}$ The growth of these Er-doped Ge-nanoparticles and their luminescence properties have been studied in detail, ${ }^{2,5,6}$ as well as the annealing behavior of highly Er-doped Ge. ${ }^{7}$ Despite the increased interest in this system, a number of fundamental questions remain unsolved. A particular one is the lattice location of the Er atoms, which largely influences the luminescence properties in semiconductors. ${ }^{8} \mathrm{In} \mathrm{Si}$, the lattice location of implanted Er has been investigated quite thoroughly. From emission channeling experiments ${ }^{9,10}$ and several first-principles calculations, ${ }^{11-13}$ the tetrahedral (T) interstitial site was found to be the preferred site in oxygenlean $\mathrm{Si}$. However, other studies suggested Er-atoms on the substitutional (S) site and the hexagonal $(\mathrm{H})$ site. $^{14,15}$ In Ge, the only study on the lattice location of rare earths is the work of Yamamoto et al., who concluded from He channeling experiments that $25 \%$ of implanted Tm occupies the $\mathrm{T}$ site. $^{16}$

\footnotetext{
${ }^{a)}$ Electronic mail: stefan.decoster@fys.kulevven.be.
}

In this letter, we present a direct lattice location study of $\mathrm{Er}$ in $\mathrm{Ge}$, measured with the emission channeling technique. ${ }^{17}$ We make use of the fact that charged particles emitted from an implanted radioactive isotope will be guided by the potential of atomic rows and planes while traveling through the crystal. The resulting anisotropic electron emission pattern around low-index crystal directions is characteristic for the lattice site occupied by the emitting atom and is measured with a two-dimensional (2D) energy- and positionsensitive Si detector of $22 \times 22$ pixels. The advantages of this technique with respect to ion beam channeling techniques, such as Rutherford Backscattering and Channeling Spectroscopy, are a considerable improvement in accuracy due to the use of 2D patterns instead of one-dimensional scans and the use of low implantation fluences, which allows us to measure the lattice location of isolated atoms.

Implantations with radioactive ${ }^{167} \mathrm{Tm}\left(t_{1 / 2}=9.25 \mathrm{~d}\right)$ were performed at room temperature at the ISOLDE facility in CERN. This isotope decays into ${ }^{167 m} \operatorname{Er}\left(t_{1 / 2}=2.27 \mathrm{~s}\right)$, emitting $\mathrm{K}, \mathrm{L}$, and $\mathrm{M}$ conversion electrons of respectively 150 , 199, and $206 \mathrm{keV}$. Three undoped Ge-samples were studied with varying implantation fluence, energy and sample orientation, and will be labeled sample A $\left(3.6 \times 10^{12} \mathrm{~cm}^{-2}\right.$, $30 \mathrm{keV},\langle 100\rangle)$, B $\left(1.1 \times 10^{13} \mathrm{~cm}^{-2}, 60 \mathrm{keV},\langle 100\rangle\right)$, and C $\left(7.7 \times 10^{12} \mathrm{~cm}^{-2}, 30 \mathrm{keV},\langle 111\rangle\right)$. To obtain unambiguous results, emission patterns around four crystal directions $(\langle 100\rangle$, $\langle 111\rangle,\langle 211\rangle$, and $\langle 110\rangle)$ were measured, analyzed consistently, and fitted to a set of simulated patterns. ${ }^{18}$ These simulations, based on the dynamical theory of electron diffraction, were performed for several high-symmetry sites such as the $\mathrm{S}, \mathrm{T}, \mathrm{H}$, bond-centered (BC), and the so-called $\mathrm{AB}, \mathrm{SP}, \mathrm{Y}$, and $\mathrm{C}$ sites, ${ }^{19}$ as well as for discrete displacements between these sites along the $\langle 111\rangle,\langle 100\rangle$, and $\langle 110\rangle$ direction. To monitor the thermal stability of the lattice location of the Er atoms, the measurements were performed after implantation as well as after annealing for $10 \mathrm{~min}$ in vacuum $\left(<10^{-5}\right.$ mbar $)$ at temperatures up to $600{ }^{\circ} \mathrm{C}$.

Figures 1(a)-1(d) show the normalized experimental electron emission patterns around the four investigated crystal directions for sample $\mathrm{C}$ after annealing at $400{ }^{\circ} \mathrm{C}$. Along the $\langle 111\rangle$-, and $\langle 100\rangle$-direction, we clearly see an enhanced number of electrons emitted along the crystal axis, while the 


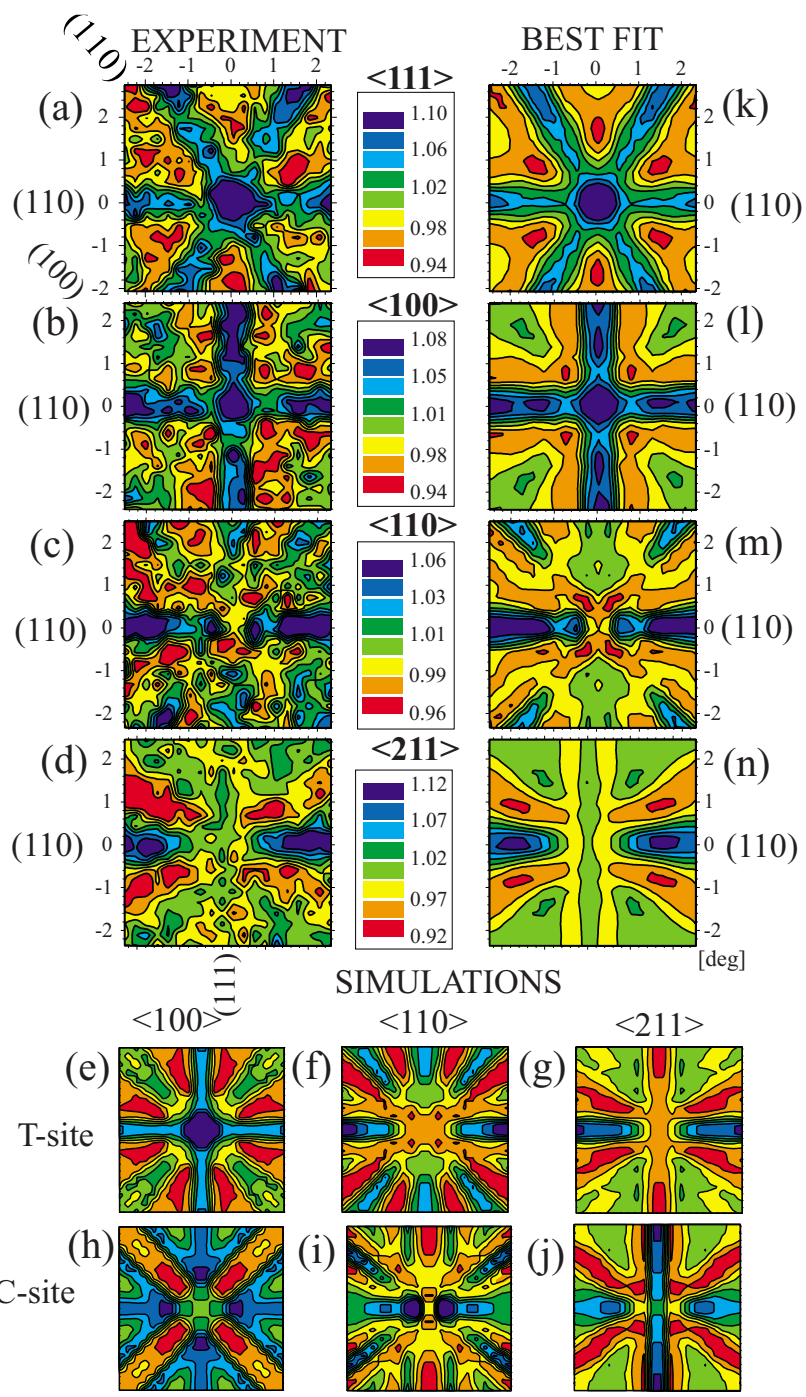

FIG. 1. (Color online) $[(\mathrm{a})-(\mathrm{d})]$ 2D conversion electron patterns emitted from ${ }^{167 m} \mathrm{Er}$ in $\mathrm{Ge}$ around the $\langle 111\rangle,\langle 100\rangle,\langle 110\rangle$, and $\langle 211\rangle$ axes, following a $400{ }^{\circ} \mathrm{C}$ annealing in vacuum. [(e)-(g)] Calculated patterns for Er-atoms occupying the $\mathrm{T}$ site and $[(\mathrm{h})-(\mathrm{j})]$ the $\mathrm{BC}$ site around the $\langle 100\rangle,\langle 110\rangle$, and $\langle 211\rangle$ axes respectively. [(k)-(n)] Best fits to the experimental patterns. The anisotropy is depicted with a color scale between red (low) and blue (high).

$\langle 110\rangle$ and $\langle 211\rangle$ patterns show a blocking effect, which is characteristic for the $\mathrm{T}$ site. However, when comparing the experimental patterns for the $\langle 100\rangle-,\langle 110\rangle$-, and $\langle 211\rangle$ direction [Figs. 1(b)-1(d)] to the corresponding calculated patterns for Er on a pure T site [Figs. 1(e)-1(g)], a number of major discrepancies are obvious. This leads to the conclusion that the Er-atoms occupy at least a second high-symmetry site. Therefore, a detailed fitting procedure was applied, considering all possible combinations of two and even three different high-symmetry sites, including possible displacements. While adding most of the high-symmetry sites $(\mathrm{H}, \mathrm{S}$, $\mathrm{SP}, \mathrm{AB}, \mathrm{Y}$, and $\mathrm{C}$ ) resulted only in marginal improvements of the fit $\left(\chi^{2}\right.$-improvement $\left.<3 \%\right)$, including a $\mathrm{BC}$ fraction results in a consistent fit in all four directions, with an average $\chi^{2}$-improvement of more than $25 \%$. Superposing the simulations for the T site $[(\mathrm{e})-(\mathrm{g})]$ and the BC site $[(\mathrm{h})-(\mathrm{j})]$, solves the discrepancies, as can be seen from Fig. 1. This is further clarified in Figs. 2(a)-2(c), showing the residual experimental pattern from Fig. 1(d) after subtracting the fit with a BC fraction only, a $\mathrm{T}$ fraction only and both $\mathrm{T}$ and $\mathrm{BC}$ fractions, respectively. Figure 2(a) clearly shows remaining features of

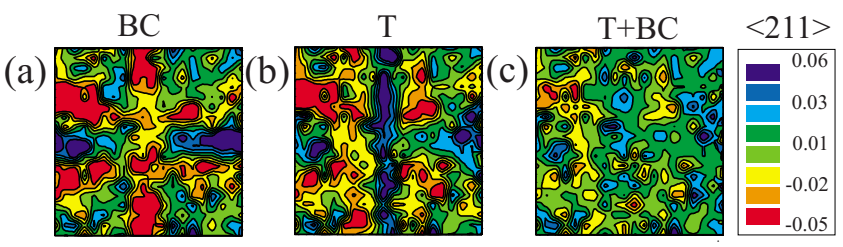

FIG. 2. (Color online) The experimental $\langle 211\rangle$-pattern from Fig. 1(d) after subtracting the best fit when allowing a fraction on (a) the $\mathrm{BC}$ site, (b) the $\mathrm{T}$ site, and (c) both $\mathrm{T}$ and $\mathrm{BC}$ sites.

a $\mathrm{T}$ site pattern, while Fig. 2(b) exhibits the same features as a BC pattern. On the other hand, Fig. 2(c) results in a featureless pattern, clearly indicating that the only satisfying fit is obtained by including a fraction on the $\mathrm{BC}$ and the $\mathrm{T}$ site. Adding a third fraction did not significantly improve the fit, indicating that only relatively small fractions $(<2 \%)$ of Er-atoms occupy other high-symmetry sites. Similar fit results have been found for the other two samples, resulting in average $\chi^{2}$ improvements between $15 \%$ and $40 \%$.

Figures $1(\mathrm{k})-1(\mathrm{n})$ show the best fit to the experimental patterns. Averaging the results of the four measured directions, $16(2) \%$ of the Er-atoms are found on the $\mathrm{BC}$ site and $20(4) \%$ on a slightly displaced T site. The Er-atoms on the T site were found to have an average root mean square displacement of about $0.18(3) \AA$, which is somewhat larger than the Ge host vibration amplitude at room temperature of $0.08 \AA$. The large remaining fraction of Er-atoms (64\%) is referred to as the random fraction. This random fraction partly consists of Er-atoms that are distributed randomly within the crystal lattice, but a large contribution will be related to the implantation-induced lattice damage. Even for the low fluences used in this study, heavy-ion implantation produces highly damaged regions, especially in materials with small lattice binding energies such as Ge. Firstly, due to the deterioration of the crystal structure, a fraction of the implanted radioactive isotopes will be located in damaged regions with reduced local crystallinity. Secondly, a fraction of the electrons emitted from an undamaged region will pass through damaged crystal regions, enhancing the probability for dechanneling. Both effects will result in an isotropic background to the patterns and consequently in the high random fraction observed in this work. However, it is important to notice that this high random fraction will not affect the qualitative analysis of the spectra.

In Fig. 3, the temperature dependence of the Er-fractions on the $\mathrm{T}$ and $\mathrm{BC}$ site is plotted for all three samples. Despite

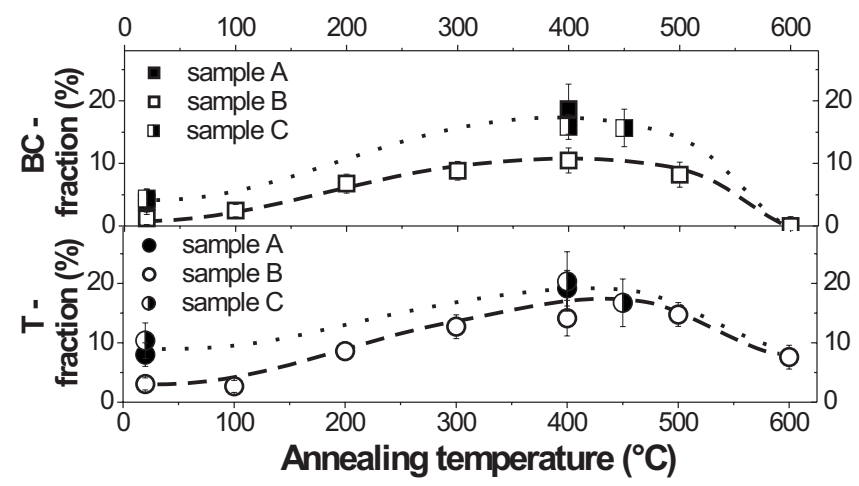

FIG. 3. Annealing temperature dependence of the fraction Er-atoms on the $\mathrm{T}$ site (circles) and the BC site (squares) for sample A (filled), B (open), and $\mathrm{C}$ (half open symbols). The dotted lines are a guide to the eye. 
the slightly different implantation conditions, the same behavior is present in all three samples. Annealing up to $400{ }^{\circ} \mathrm{C}$ results in an increasing fraction of Er-atoms on highsymmetry sites, which is a direct consequence of the partial lattice recovery of damaged regions. This fraction decreases again after annealing at temperatures higher than $450{ }^{\circ} \mathrm{C}$, which is most likely related to diffusion of the Er-atoms. As the depth profile of the implanted Er-atoms is an input parameter for the simulations, it implies that a larger mean projected range of the Er-atoms after diffusion will lead to an underestimation of the measured and fitted fraction on highsymmetry sites. Alternatively, the trapping of Er-atoms by migrating defects may result in Er-atoms occupying low symmetry sites in defect complexes.

The existence of tetrahedral interstitial $\mathrm{Er}$ in $\mathrm{Si}$ has been proven both from experiments and calculations. Due to the similar lattice structure of $\mathrm{Si}$ and $\mathrm{Ge}, \mathrm{Er}$ can be expected to occupy the $\mathrm{T}$ site in $\mathrm{Ge}$ as well, which was found experimentally in this study. So far, there is no clear explanation for the small displacement of the Er-atoms on the T site. However, it could be originating from lattice relaxations as was predicted from density functional computations. ${ }^{14}$ Applying stress related arguments, Er is not expected to occupy a BC site in an undamaged Ge crystal. However, we believe this site is related to an Er-vacancy (Er-V) complex. From ab initio density functional calculations, a range of high- $Z$ impurities (from $\mathrm{Cd}$ to $\mathrm{Bi}$ ) were found to be unstable on the $\mathrm{S}$ site with a vacancy on the nearest neighbor $\mathrm{S}$ site. ${ }^{20}$ The impurity prefers to shift towards the BC site, with the vacancy split between the two sites. Ion implantation is known to produce many vacancies and interstitials, which are mobile at room temperature in $\mathrm{Ge}^{21}$ This implies that during the implantation, they are free to migrate through the crystal until they either recombine, form complex clusters or get trapped by impurity atoms. Although no calculations have been performed for Er, interpolating the trend that high- $Z$ impurities are stable on the $\mathrm{BC}$ site, allows us to conclude that our experimental observation of Er on the BC site in Ge might be indirect evidence of the Er-V complex in the split-vacancy configuration. It can not be excluded that other (more complex) defect configurations may also contribute to the observed bond-centered behavior.

In conclusion, we have found experimental evidence of implanted Er atoms located on the T site and on the BC site in Ge. While the occupation of the T site could be expected from comparison with similar studies in $\mathrm{Si}$, this study offers direct evidence of the occupation of the $\mathrm{BC}$ site in Ge. This site has been predicted by $a b$ initio density functional calculations for heavy impurities in Ge and we believe it is related to the Er-V complex in the split-vacancy configuration. Since the luminescent properties of dopants are significantly influenced by their lattice site, the observation of Er on two different sites can be of great importance in understandingand even tuning - the optical properties of the Ge:Er system.

This work was supported by FWO Flanders (G.0501.07 and G.0636.08), the K.U. Leuven projects GOA/2004/02 and INPAC EF/05/2005, the IUAP P6/42 programme, the Portuguese Foundation for Science and Technology (POCI-FP81921-2007) and the ISOLDE collaboration. S.D. acknowledges financial support from FWO, Flanders.

${ }^{1}$ A. Kenyon, Semicond. Sci. Technol. 20, R65 (2005).

${ }^{2}$ S. Sato, S. Nozaki, H. Morisaki, and M. Iwase, Appl. Phys. Lett. 66, 3176 (1995).

${ }^{3}$ C. Bostedt, T. van Buuren, T. Willey, N. Franco, L. Terminello, C. Heske, and T. Moller, Appl. Phys. Lett. 84, 4056 (2004).

${ }^{4}$ M. Jing, M. Ni, W. Song, J. Lu, Z. Gao, L. Lai, W. Mei, D. Yu, H. Ye, and L. Wang, J. Phys. Chem. B 110, 18332 (2006).

${ }^{5}$ C. Heng, T. Finstad, P. Storas, Y. Li, A. Gunnaes, and O. Nilsen, Appl. Phys. Lett. 85, 4475 (2004).

${ }^{6}$ J. Chen, D. Pang, H. Cheong, P. Wickboldt, and W. Paul, Appl. Phys. Lett. 67, 2182 (1995).

${ }^{7}$ S. Kucheyev, J. Bradby, S. Ruffell, C. Li, T. Felter, and A. Hamza, Appl. Phys. Lett. 90, 221901 (2007).

${ }^{8}$ Y. Tang, K. Heasman, W. Gillin, and B. Sealy, Appl. Phys. Lett. 55, 432 (1989)

${ }^{9}$ U. Wahl, A. Vantomme, J. De Wachter, R. Moons, G. Langouche, J. Marques, J. Correia, and ISOLDE Collaboration, Phys. Rev. Lett. 79, 2069 (1997)

${ }^{10}$ U. Wahl, J. Correia, J. Araújo, A. Vantomme, G. Langouche, and ISOLDE Collaboration, Physica B 273, 342 (1999).

${ }^{11}$ M. Needels, M. Schluter, and M. Lannoo, Phys. Rev. B 47, 15533 (1993)

${ }^{12}$ Y. Wan, Y. Ling, Q. Sun, and X. Wang, Phys. Rev. B 58, 10415 (1998).

${ }^{13}$ D. Prezzi, T. Eberlein, R. Jones, J. Filhol, J. Coutinho, M. Shaw, and P. Briddon, Phys. Rev. B 71, 245203 (2005).

${ }^{14}$ A. Raffa and P. Ballone, Phys. Rev. B 65, 121309 (2002).

${ }^{15}$ A. Kozanecki, R. Wilson, B. Sealy, J. Kaczanowski, and L. Nowicki, Appl. Phys. Lett. 67, 1847 (1995).

${ }^{16}$ Y. Yamamoto, M. Wakaiki, A. Ikeda, and Y. Kido, Nucl. Instrum. Methods Phys. Res. B 153, 452 (1999).

${ }^{17}$ U. Wahl, J. Correia, A. Czermak, S. Jahn, P. Jalocha, J. Marques, A. Rudge, F. Schopper, J. Soares, A. Vantomme et al., Nucl. Instrum. Methods Phys. Res. A 524, 245 (2004).

${ }^{18}$ H. Hofsäss and G. Lindner, Phys. Rep. 201, 121 (1991).

${ }^{19}$ U. Wahl, Phys. Rep. 280, 145 (1997).

${ }^{20}$ H. Höhler, N. Atodiresei, K. Schroeder, R. Zeller, and P. Dederichs, Phys Rev. B 71, 035212 (2005).

${ }^{21}$ H. Hässlein, R. Sielemann, and C. Zistl, Phys. Rev. Lett. 80, 2626 (1998). 